\title{
Metoprolol treatment for two years after coronary bypass grafting: effects on exercise capacity and signs of myocardial ischaemia
}

\author{
Helén Sjöland, Kenneth Caidahl, Leon Lurje, Åke Hjalmarson, Johan Herlitz
}

\begin{abstract}
Objective-To evaluate whether prophylactic treatment with metoprolol for two years after coronary artery bypass grafting improves working capacity and reduces the occurrence of myocardial ischaemia in patients with coronary artery disease.

Methods-After coronary artery bypass grafting, patients were randomised to treatment with metoprolol or placebo for two years. Two years after randomisation, a computerised 12-lead electrocardiogram was obtained during a standardised bicycle exercise test in 618 patients (64\% of all those randomised). Results-The median exercise capacity was $140 \mathrm{~W}$ in the metoprolol group ( $n=$ $307)$ and $130 \mathrm{~W}$ in the placebo group $(\mathrm{n}=$ 311) $(P>0 \cdot 20)$. An ST depression of $\geqslant 1$ mm at maximum exercise was present in $34 \%$ of the patients in the metoprolol group and $38 \%$ in the placebo group (P > 0.20) and an ST depression of $\geqslant 2 \mathrm{~mm}$ at maximum exercise was present in $11 \%$ in the metoprolol group and $16 \%$ in the placebo group $(P=0.09)$. The median values for maximum systolic blood pressure were $200 \mathrm{~mm} \mathrm{Hg}$ in the metoprolol group and $210 \mathrm{~mm} \mathrm{Hg}$ in the placebo group ( $P<0.0001)$, while the median values for maximum heart rate were 126 beats/min in the metoprolol group and 143 beats/min in the placebo group $(P<$ $0.0001)$. The occurrence of cardiac and neurological clinical events two years postoperatively among exercised patients was comparable in the treatment groups. Conclusions-Treatment with metoprolol for two years after coronary artery bypass grafting did not significantly change exercise capacity or electrocardiographic signs of myocardial ischaemia.
\end{abstract}

(Br Heart f 1995;74:235-241)

Keywords: metoprolol; coronary bypass grafting; exercise capacity; myocardial ischaemia

Coronary artery bypass grafting (CABG) is a frequent and costly procedure in coronary artery disease, and is performed with the primary objective of relieving pain and improving survival. ${ }^{12}$ The underlying atherosclerotic disease remains, ${ }^{3}$ and the recurrence of symptoms is common after several years. ${ }^{4}$ The return of angina pectoris in the increasing population of patients who have undergone CABG calls for medication for angina and possibly subsequent revascularisation. It is therefore important to find a means of decreasing the reappearance of angina pectoris. Animal studies have indicated that atherosclerosis decreases during treatment with metoprolol, ${ }^{5}$ a selective $\beta$ adrenoceptor blocking agent. In a consecutive series of patients who underwent CABG, we performed a double blind randomised study to evaluate the effect of metoprolol on the development of ischaemic events, exercise capacity, and signs of myocardial ischaemia two years after CABG. Working capacity and signs of exercise induced ischaemia were evaluated in a bicycle exercise test two years after CABG in the metoprolol and placebo groups. The occurrence of ischaemic events in the exercised patients was studied. The aim of the present study was to evaluate whether treatment with metoprolol for two years after CABG improves exercise capacity and reduces signs of myocardial ischaemia.

\section{Methods}

PATIENTS

All the patients from western Sweden who underwent CABG at Sahlgrenska University Hospital and the Scandinavian Heart Centre, Göteborg, between June 1988 and June 1991 were evaluated for inclusion in a double blind randomised study of the anti-ischaemic effects of metoprolol after bypass surgery during two years of treatment. Between four and 21 days after CABG, patients were randomised to receive $100 \mathrm{mg}$ of metoprolol every day for two weeks and thereafter $200 \mathrm{mg}$ of metoprolol every day for two years, or placebo. Patients were recruited from all 15 hospitals in the south western region of Sweden. During the study period, 2365 patients underwent CABG, of whom $967(41 \%)$ were included in the metoprolol study. The exclusion criteria were simultaneous valve surgery $(n=261,19 \%)$, unwillingness or inability to give informed consent $(n=254,18 \%)$, need for open treatment with $\beta$ blockade $(n=194,14 \%)$, age $>75$ years $(n=170,12 \%)$, systolic blood pressure $<100 \mathrm{~mm} \mathrm{Hg}(\mathrm{n}=57,4 \%)$, severe obstructive pulmonary disease $(n=62,4 \%)$, participation in other randomised trials $(n=61$, $4 \%)$, and death $(n=42,3 \%)$. Eighty seven patients $(6 \%)$ were excluded due to a heart rate of $<45$ beats/min, severe heart failure, poor peripheral circulation, advanced atrioventricular block, or previous participation in 
the study. Three hundred and eighty seven patients $(28 \%)$ were excluded for other reasons. Among these, 74 patients ( $8 \%$ of all) were never evaluated for inclusion before their death. Frequent conditions registered as other reasons included: the patient reported intolerance to $\beta$ blockade, though not confirmed or assessed as a contraindication by the treating physician; the patient was unwilling to discontinue medication with metoprolol after CABG; and reported hypertension where $\beta$ blockade was approved by the patient and the physician. More than one reason for exclusion may have been recorded for each patient. Two years after CABG, the trial protocol specified that all randomised patients were summoned for exercise testing at the referring hospital. A two year exercise test was performed by 618 $(64 \%)$ of the study participants. No record of omission was made. Two of the participating hospitals were unable to carry out systematic two year exercise testing - accounting for the omission of 148 patients (15\%)-for administrative reasons. The patients who actually participated in exercise tests were evenly distributed between the treatment groups. Angina functional status was classified as follows: functional class 1 , no symptoms of angina pectoris; functional class 2 , angina on heavy effort; functional class 3 , angina on moderate or light effort; functional class 4, angina on very light effort or at rest.

\section{EXERCISE TESTING}

Exercise tests were performed at Sahlgrenska University Hospital or at the referring hospital. The exercise tests were performed in a standardised manner with a 12-lead electrocardiogram (ECG) on a bicycle ergometer. Supine and sitting measurements were recorded before the start of the upright exercise. The exercise was started at $30 \mathrm{~W}$ for women and $50 \mathrm{~W}$ for men with $10 \mathrm{~W}$ increments every minute. Four different computer aided electrocardiographic systems or analogous techniques were used: the $T$ Bjurö system, ${ }^{6}$ the Swedish system, ${ }^{7}$ the Schiller system, and the Marquette system; 47 patients $(8 \%)$ performed an analogous test. In 10 cases (2\%), the exercise tests were computer aided but measurements were made manually for technical reasons. A record of the technique was missing in five cases $(0.8 \%)$. All data in this study were recorded in a standardised protocol. Blood pressure and perceived effort, chest pain, and dyspnoea on a 10 degree Borg scale ${ }^{8}$ were noted for each load. Heart rate and electrocardiographic changes were either continuously monitored or, in the case of the analogous exercise tests, recorded at each load. During exercise, a 12-lead ECG was recorded with computerised systems and in case analogous systems were used a precordial ECG was made. The termination criteria were: perceived maximum effort or dyspnoea, severe chest pain, severe signs of myocardial ischaemia, severe arrhythmias, extreme hypertensive response, or hypotension at increasing work level. Maximum chest pain, dyspnoea, and effort were recorded at the end of exercise.
The end of the electrocardiographic PR segment was used as the reference point for ST measurements and the ST amplitude 60 ms after the J point was used as the ST level. ST amplitude changes were recorded in leads aVF, V2, and V5 in the study protocol..$^{910}$ The exercise test protocol specified an assessment of myocardial ischaemia based on the ECG reaction: a horizontal or downsloping ST segment depression of $1 \mathrm{~mm}$ or more was regarded as a positive ECG reaction for myocardial ischaemia if it occurred during maximum exercise or within four minutes after exercise.

The test protocol also specified a physician's comprehensive assessment of the exercise test, which was performed by one of the investigators (HS) blinded to the clinical history. The physician's assessment was made according to the following classification. Definite myocardial ischaemia: The ECG showed changes consistent with the definition of exercise induced myocardial ischaemia at maximum exercise or four minutes after. The resting ECG was normal, or without confounding abnormalities such as bundle branch block, ST segment depression at rest, $Q$ wave, or QS complex. Probable myocardial ischaemia: The clinical appearance was consistent with exercise induced myocardial ischaemia but the ECG criteria were questionable. For example: the patient revealed chest pain consistent with angina pectoris in the presence of bundle branch block, pacemaker ECG, $Q$ wave, QS complex, or considerable ST depression in the resting ECG. Indeterminate: The exercise ECG did not reveal enough information either to confirm or rule out myocardial ischaemia. No ischaemia: The test revealed no chest pain or ECG changes consistent with myocardial ischaemia. Tests were also regarded as positive by the physician in cases in which the significant ST segment depression occurred in a lead that was not recorded in the study protocol, since the physician's assessment was based on ST amplitude changes in all recorded leads, and the complete exercise ECG was available for the investigator at the time of assessment. ST segment measurements were only used in the statistical analysis when measurements were recorded in all three leads in the protocol. For example, when evaluating ST segment depression $\geqslant 1 \mathrm{~mm}$, we required that readings were submitted to us from all three leads (aVF, V2, and V5). The number of observations noted in the tables differs due to incomplete data in the study protocols. Most analogous ECGs registered only precordial leads during exercise, and were thus not included in this analysis.

STATISTICAL ANALYSIS

Fisher's permutation test, including Fisher's exact test for dichotomous variables, was used to test for differences between groups. In the tables, $\mathbf{P}$ values are only given when they are below $0 \cdot 05$. All $P$ values are two tailed and, in line with practice, no corrections for multiple comparisons have been made. Therefore, $P$ 
values of borderline significance $(P<0.05)$ should be regarded with caution.

\section{Results}

Of all the patients who underwent CABG during the study period, 967 participated in the metoprolol trial. Of these, 618 performed

Table 1 Clinical characteristics at operation

\begin{tabular}{|c|c|c|c|c|c|}
\hline & \multicolumn{2}{|c|}{ Placebo } & \multicolumn{2}{|c|}{ Metoprolol } & \multirow[b]{2}{*}{$P$ value } \\
\hline & $n$ & $\%$ & $n$ & $\%$ & \\
\hline $\begin{array}{l}\text { Number of patients } \\
\text { Men } \\
\text { Women } \\
\text { Age } \geqslant 65 \text { years } \\
\text { Age }<65 \text { years }\end{array}$ & $\begin{array}{r}311 \\
273 \\
38 \\
147 \\
164\end{array}$ & $\begin{array}{l}88 \\
12 \\
47 \\
53\end{array}$ & $\begin{array}{r}307 \\
262 \\
45 \\
116 \\
191\end{array}$ & $\begin{array}{l}85 \\
15 \\
38 \\
62\end{array}$ & \} $0.02^{\star}$ \\
\hline $\begin{array}{l}\text { History of } \\
\text { Angina pectoris } \\
\text { Myocardial infarction } \\
\text { Congestive heart failure } \\
\text { Hypertension } \\
\text { Diabetes mellitus } \\
\text { Claudication } \\
\text { Cerebrovascular disease } \\
\text { Smoking } \\
\text { Previous smoking }\end{array}$ & $\begin{array}{r}307 \\
179 \\
35 \\
91 \\
37 \\
34 \\
19 \\
31 \\
201\end{array}$ & $\begin{array}{r}99 \\
58 \\
11 \\
29 \\
12 \\
11 \\
6 \\
10 \\
65\end{array}$ & $\begin{array}{r}301 \\
164 \\
27 \\
104 \\
34 \\
31 \\
17 \\
34 \\
185\end{array}$ & $\begin{array}{r}98 \\
53 \\
9 \\
34 \\
11 \\
10 \\
6 \\
11 \\
60\end{array}$ & \\
\hline $\begin{array}{l}\text { Angina functional class }(8)^{a} \\
\quad 1 \\
2 \\
3 \\
4\end{array}$ & $\begin{array}{r}4 \\
45 \\
180 \\
79\end{array}$ & $\begin{array}{r}1 \\
15 \\
58 \\
26\end{array}$ & $\begin{array}{r}6 \\
49 \\
171 \\
76\end{array}$ & $\begin{array}{r}2 \\
16 \\
57 \\
25\end{array}$ & \\
\hline $\begin{array}{l}\text { Medication at discharge } \\
\text { Calcium antagonists } \\
\text { Long acting nitrates } \\
\text { Diuretics for heart failure } \\
\text { Digitalis } \\
\text { Other treatment for heart failure } \\
\text { Antihypertensives } \\
\text { Antiarrhythmics } \\
\text { Acetylsalicylic acid } \\
\text { Anticoagulation }\end{array}$ & $\begin{array}{r}13 \\
4 \\
53 \\
95 \\
7 \\
8 \\
28 \\
287 \\
12\end{array}$ & $\begin{array}{r}4 \\
1 \\
17 \\
31 \\
2 \\
3 \\
9 \\
93 \\
4\end{array}$ & $\begin{array}{r}18 \\
1 \\
40 \\
82 \\
4 \\
17 \\
24 \\
280 \\
15\end{array}$ & $\begin{array}{c}6 \\
0 \cdot 3 \\
13 \\
27 \\
1 \\
6 \\
8 \\
92 \\
5\end{array}$ & \\
\hline
\end{tabular}

${ }^{\star} P$ value refers to age groups (for the overall age distribution $P=0.09$ ).

${ }^{a}$ Number in parentheses denotes missing patients.

Table 2 Characteristics at operation for participants/non-participants in exercise tests two years after coronary artery bypass grafting

\begin{tabular}{|c|c|c|c|c|c|}
\hline & \multicolumn{2}{|c|}{$\begin{array}{l}\text { No two year } \\
\text { exercise test }\end{array}$} & \multicolumn{2}{|c|}{$\begin{array}{l}\text { Two year } \\
\text { exercise test }\end{array}$} & \multirow[b]{2}{*}{$P$ value } \\
\hline & $n$ & $\%$ & $n$ & $\%$ & \\
\hline $\begin{array}{l}\text { Number of patients } \\
\text { Men } \\
\text { Women } \\
\text { Age } \geqslant 65 \text { years } \\
\text { Age }<65 \text { years }\end{array}$ & $\begin{array}{r}349 \\
291 \\
58 \\
181 \\
168\end{array}$ & $\begin{array}{l}83 \\
17 \\
52 \\
48\end{array}$ & $\begin{array}{r}618 \\
535 \\
83 \\
263 \\
355\end{array}$ & $\begin{array}{l}87 \\
13 \\
43 \\
57\end{array}$ & \} $0.006^{\star}$ \\
\hline $\begin{array}{l}\text { History of } \\
\text { Angina pectoris } \\
\text { Myocardial infarction } \\
\text { Congestive heart failure } \\
\text { Hypertension } \\
\text { Diabetes mellitus } \\
\text { Claudication } \\
\text { Cerebrovascular disease } \\
\text { Smoking } \\
\text { Previous smoking }\end{array}$ & $\begin{array}{r}341 \\
215 \\
67 \\
139 \\
44 \\
40 \\
32 \\
48 \\
206\end{array}$ & $\begin{array}{r}98 \\
62 \\
19 \\
40 \\
13 \\
11 \\
9 \\
14 \\
59\end{array}$ & $\begin{array}{r}608 \\
343 \\
62 \\
195 \\
71 \\
65 \\
36 \\
65 \\
386\end{array}$ & $\begin{array}{r}98 \\
56 \\
10 \\
32 \\
11 \\
11 \\
6 \\
11 \\
62\end{array}$ & $\begin{array}{c}<0.0001 \\
0.009\end{array}$ \\
\hline $\begin{array}{l}\text { Angina functional class }(14)^{a} \\
\quad 1 \\
2 \\
3 \\
4\end{array}$ & $\begin{array}{r}8 \\
24 \\
203 \\
108\end{array}$ & $\begin{array}{r}2 \\
7 \\
59 \\
31\end{array}$ & $\begin{array}{r}10 \\
94 \\
351 \\
155\end{array}$ & $\begin{array}{r}2 \\
15 \\
58 \\
25\end{array}$ & 0.005 \\
\hline $\begin{array}{l}\text { Medication at discharge } \\
\text { Calcium antagonists } \\
\text { Long acting nitrates } \\
\text { Diuretics for heart failure } \\
\text { Digitalis } \\
\text { Other treatment for heart failure } \\
\text { Antihypertensives } \\
\text { Antiarrhythmics } \\
\text { Acetylsalicylic acid } \\
\text { Anticoagulation }\end{array}$ & $\begin{array}{r}26 \\
2 \\
92 \\
107 \\
10 \\
21 \\
22 \\
317 \\
23\end{array}$ & $\begin{array}{c}8 \\
0 \cdot 6 \\
27 \\
31 \\
3 \\
6 \\
6 \\
92 \\
7\end{array}$ & $\begin{array}{r}31 \\
5 \\
93 \\
177 \\
11 \\
25 \\
52 \\
567 \\
27\end{array}$ & $\begin{array}{c}5 \\
0 \cdot 8 \\
15 \\
29 \\
2 \\
4 \\
8 \\
92 \\
4\end{array}$ & $<0.0001$ \\
\hline
\end{tabular}

an exercise test two years after CABG and are included in the present analysis. This constituted $64 \%$ of the patients in the metoprolol trial and $26 \%$ of all the patients operated on during the study period. One hundred and ten patients in the placebo group and 83 patients in the metoprolol group had been withdrawn from blind medication before the time of the postoperative exercise ECG.

BASELINE CHARACTERISTICS (tables 1 and 2)

The metoprolol and placebo groups were comparable in terms of clinical characteristics at operation. More patients in the metoprolol group were under 65 years of age, but the difference in age was not significant for the overall age distribution. Patients who did not perform the two year exercise test follow up suffered from more severe heart disease than those who participated. They had a higher prevalence of congestive heart failure, diuretic treatment, hypertension, and more advanced anginal class, and they were older than the patients who took part in the exercise tests.

WORKING CAPACITY (fig 1)

The median values for maximum exercise capacity were $140 \mathrm{~W}$ in the metoprolol group and $130 \mathrm{~W}$ in the placebo group. Among males, the median values for maximum exercise capacity were $140 \mathrm{~W}$ in both groups and the corresponding figures among females were $90 \mathrm{~W}$ in both groups.

BLOOD PRESSURE AND HEART RATE (table 3)

There was a significantly lower median systolic blood pressure and heart rate in the metoprolol group at $70 \mathrm{~W}$, at maximum exercise, and four minutes after exercise as compared to placebo. The resting diastolic blood pressure and heart rate were also found to be lower in the metoprolol group. The numbers of observations differ because of incomplete data in the study protocols.

ST SEGMENT DEPRESSION, PAIN, AND ISCHAEMIA (tables 4 and 5)

Based on ST segment criteria ( $\geqslant 1 \mathrm{~mm}$ ST segment depression), myocardial ischaemia was present, at maximum exercise and four

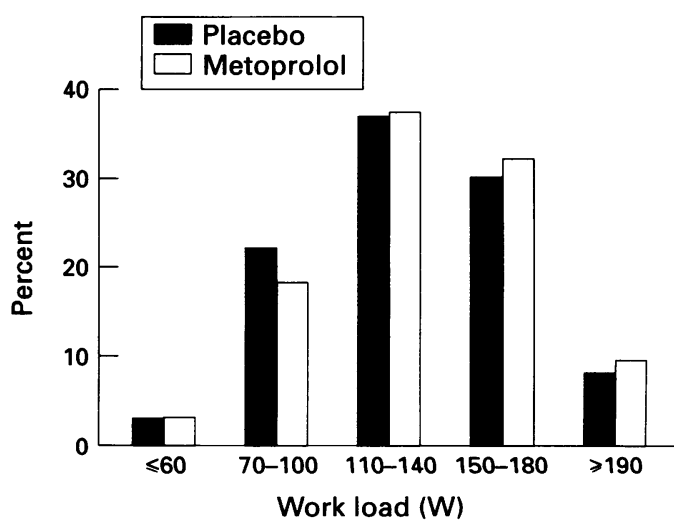

Figure 1 Exercise capacity two years after coronary artery bypass grafting in metoprolol $(n=306)$ and placebo $(n=311)$ groups 
minutes after, in a greater proportion of the placebo patients compared to the metoprolol group, although the difference was not significant. Definite or probable myocardial ischaemia, by the investigator's assessment, was adjudged to be present in $33 \%$ of patients in the placebo group and $27 \%$ in the metoprolol group $(P=0 \cdot 11)$. There was no significant difference between the metoprolol and placebo groups with respect to the proportion

Table 3 Blood pressure and heart rate during exercise two years after coronary artery bypass grafting

\begin{tabular}{|c|c|c|c|c|c|}
\hline & \multicolumn{2}{|c|}{ Placebo } & \multicolumn{2}{|c|}{ Metoprolol } & \multirow[b]{2}{*}{$P$ value } \\
\hline & $n$ & Median & $n$ & Median & \\
\hline \multicolumn{6}{|l|}{$\begin{array}{l}\text { Systolic blood pressure } \\
(\mathrm{mm} \mathrm{Hg})\end{array}$} \\
\hline $\begin{array}{l}\text { Baseline } \\
\text { At } 70 \mathrm{~W} \\
\text { At maximum exercise } \\
\text { At } 4 \text { min post exercise }\end{array}$ & $\begin{array}{l}302 \\
273 \\
272 \\
285\end{array}$ & $\begin{array}{l}150 \\
180 \\
210 \\
162\end{array}$ & $\begin{array}{l}285 \\
252 \\
259 \\
263\end{array}$ & $\begin{array}{l}145 \\
170 \\
200 \\
158\end{array}$ & $\begin{aligned}< & 0.0001 \\
< & 0.0001 \\
& 0.02\end{aligned}$ \\
\hline \multicolumn{6}{|l|}{$\begin{array}{l}\text { Diastolic blood pressure } \\
(\mathrm{mm} \mathrm{Hg})\end{array}$} \\
\hline Baseline & 310 & 85 & 303 & 84 & $<0.004$ \\
\hline \multicolumn{6}{|l|}{ Heart rate (beats/min) } \\
\hline $\begin{array}{l}\text { Baseline } \\
\text { At } 70 \mathrm{~W} \\
\text { At maximum exercise }\end{array}$ & $\begin{array}{l}308 \\
299 \\
306\end{array}$ & $\begin{array}{r}71 \\
106 \\
143\end{array}$ & $\begin{array}{l}299 \\
289 \\
305\end{array}$ & $\begin{array}{r}62 \\
92 \\
126\end{array}$ & $\begin{array}{l}<0.0001 \\
<0.0001 \\
<0.0001\end{array}$ \\
\hline At $4 \mathrm{~min}$ post exercise & 304 & 92 & 297 & 80 & $<0.0001$ \\
\hline
\end{tabular}

Table 4 ST level changes, pain, and ischaemia at exercise two years after coronary artery bypass grafting

\begin{tabular}{|c|c|c|c|c|c|}
\hline & \multicolumn{2}{|c|}{ Placebo } & \multicolumn{2}{|c|}{ Metoprolol } & \multirow[b]{2}{*}{ Pvalue } \\
\hline & $n$ & $\%$ & $n$ & $\%$ & \\
\hline $\begin{array}{l}\text { At } 70 \mathrm{~W} \\
\text { ST depression } \geqslant 1 \mathrm{~mm} \\
\text { ST depression } \geqslant 2 \mathrm{~mm}\end{array}$ & $\begin{array}{r}248 \\
29 \\
7\end{array}$ & $\begin{array}{r}12 \\
3\end{array}$ & $\begin{array}{r}244 \\
33 \\
3\end{array}$ & $\begin{array}{r}14 \\
1\end{array}$ & \\
\hline $\begin{array}{l}\text { At maximum exercise } \\
\text { ST depression } \geqslant 1 \mathrm{~mm} \\
\text { ST depression } \geqslant 2 \mathrm{~mm}\end{array}$ & $\begin{array}{r}283 \\
108 \\
45\end{array}$ & $\begin{array}{l}38 \\
16\end{array}$ & $\begin{array}{r}284 \\
96 \\
31\end{array}$ & $\begin{array}{l}34 \\
11\end{array}$ & \\
\hline $\begin{array}{l}\text { At } 4 \mathrm{~min} \text { postexercise } \\
\text { ST depression } \geqslant 1 \mathrm{~mm} \\
\text { ST depression } \geqslant 2 \mathrm{~mm}\end{array}$ & $\begin{array}{r}282 \\
60 \\
17\end{array}$ & $\begin{array}{r}21 \\
6\end{array}$ & $\begin{array}{r}287 \\
50 \\
12\end{array}$ & $\begin{array}{r}17 \\
4\end{array}$ & \\
\hline $\begin{array}{l}\text { Exercise test assessment } \\
\text { Definite myocardial ischaemia } \\
\text { Probable myocardial ischaemia } \\
\text { Indeterminate } \\
\text { No ischaemia }\end{array}$ & $\begin{array}{r}309 \\
49 \\
54 \\
40 \\
166\end{array}$ & $\begin{array}{l}16 \\
17 \\
13 \\
54\end{array}$ & $\begin{array}{r}305 \\
37 \\
46 \\
46 \\
176\end{array}$ & $\begin{array}{l}12 \\
15 \\
15 \\
58\end{array}$ & \\
\hline $\begin{array}{l}\text { Chest pain assessed } \\
\text { Angina pectoris }\end{array}$ & $\begin{array}{r}311 \\
33\end{array}$ & 11 & $\begin{array}{r}306 \\
48\end{array}$ & 16 & \\
\hline $\begin{array}{l}\text { Estimation of subjective symptoms } \\
\text { Effort }(1-10) \\
\text { Dyspnoea }(0-10) \\
\text { Chest pain }(0-10)\end{array}$ & $\begin{array}{r}\text { Mean } \\
7 \cdot 4 \\
6 \cdot 5 \\
0 \cdot 6\end{array}$ & $\begin{array}{l}(\text { SEM) } \\
(0 \cdot 1) \\
(0 \cdot 1) \\
(0 \cdot 1)\end{array}$ & $\begin{array}{r}\text { Mean } \\
7 \cdot 6 \\
6 \cdot 6 \\
1 \cdot 1\end{array}$ & $\begin{array}{l}(\text { SEM) } \\
(0 \cdot 1) \\
(0 \cdot 1) \\
(0 \cdot 1)\end{array}$ & 0.001 \\
\hline
\end{tabular}

Table 5 Findings at exercise according to actual treatment two years after coronary artery bypass grafting

\begin{tabular}{|c|c|c|c|c|c|}
\hline \multirow{2}{*}{$\begin{array}{l}\text { Exercise capacity } \\
\text { (W, median) }\end{array}$} & \multicolumn{2}{|c|}{$\begin{array}{l}\text { Not on } \beta \text { blockade } \\
(n=287)\end{array}$} & \multicolumn{2}{|c|}{$\begin{array}{l}\text { On } \beta \text { blockade } \\
(n=325)\end{array}$} & \multirow{2}{*}{$\frac{P \text { value }}{0.02}$} \\
\hline & 140 & & 130 & & \\
\hline & \multicolumn{4}{|c|}{$S T$ segment depression at exercise } & \\
\hline $\begin{array}{l}\text { At } 70 \mathrm{~W} \\
\text { ST depression } \geqslant 1 \mathrm{~mm} \\
\text { ST depression } \geqslant 1 \mathrm{~mm}\end{array}$ & $\begin{array}{r}228 \\
25 \\
7\end{array}$ & $\begin{array}{r}11 \\
3\end{array}$ & $\begin{array}{r}260 \\
35 \\
3\end{array}$ & $\begin{array}{r}13 \\
1\end{array}$ & \\
\hline $\begin{array}{l}\text { At maximum exercise } \\
\text { ST depression } \geqslant 1 \mathrm{~mm} \\
\text { ST depression } \geqslant 1 \mathrm{~mm}\end{array}$ & $\begin{array}{r}260 \\
95 \\
40\end{array}$ & $\begin{array}{l}37 \\
15\end{array}$ & $\begin{array}{r}301 \\
107 \\
34\end{array}$ & $\begin{array}{l}36 \\
11\end{array}$ & \\
\hline $\begin{array}{l}\text { At } 4 \mathrm{~min} \text { postexercise } \\
\text { ST depression } \geqslant 1 \mathrm{~mm} \\
\text { ST depression } \geqslant 1 \mathrm{~mm}\end{array}$ & $\begin{array}{r}258 \\
44 \\
13\end{array}$ & $\begin{array}{r}17 \\
5\end{array}$ & $\begin{array}{r}305 \\
64 \\
15\end{array}$ & $\begin{array}{r}21 \\
5\end{array}$ & \\
\hline
\end{tabular}

with angina pectoris during exercise, $16 \%$ versus $11 \%(P=0.07)$. The mean chest pain score was significantly higher in the metoprolol group than in the placebo group ( $P=0.001)$. When the groups were analysed in terms of actual treatment, we found a lower exercise capacity in the group treated with any kind of $\beta$ blockade $(P=0.02)$, but no significant difference in the incidence and severity of ST depression during exercise.

\section{IMPROVEMENT IN EXERCISE PERFORMANCE}

(fig 2)

There was no difference between the groups with respect to improvement in exercise capacity. The median improvement in exercise capacity was $30 \mathrm{~W}$ in both treatment groups. The results were similar among patients still on blind medication at the time of exercise and among patients who had stopped blind treatment prematurely.

FURTHER OBSERVATIONS (table 6)

The prevalence and distribution of reasons for terminating exercise did not differ between the groups. Half the patients in both treatment groups therefore stopped the exercise because of general tiredness. This was followed, in order of frequency, by leg tiredness, dyspnoea, and chest pain, which occurred with a similar frequency in the two groups.

The proportion of patients with any ventricular premature beats during exercise was considerable in both treatment groups, although it was significantly more common in the placebo group ( $P=0.005)$, just exceeding half of all exercised patients, as compared with $41 \%$ in the metoprolol group. The occurrence of higher numbers of ventricular premature beats $(>5 / \mathrm{min}$ ) was also more common in the placebo group, although the significance was weaker $(P=0.04)$. Withdrawal from treatment was more common in the placebo group than in the metoprolol group, at $35 \%$ versus $27 \%(P=0.03)$. Open $\beta$ blockade treatment was also significantly more common in the placebo group ( $P=0.004)$, as was treatment with digitalis $(\mathbf{P}=0.006)$.

CLINICAL EVENTS AND REASONS FOR

WITHDRAWAL (table 7)

There was no significant difference in the clinical event rate between the two treatment

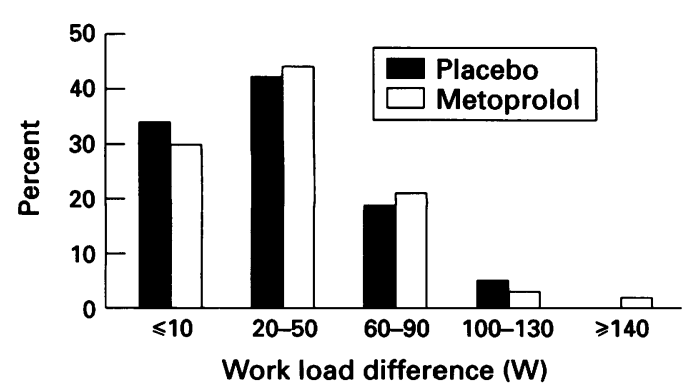

Figure 2 Improvement in exercise capacity two years after coronary artery bypass grafting compared with preoperative exercise test results in metoprolol $(n=183)$ and placebo $(n=178)$ groups 
groups during two years after CABG, with respect to myocardial infarction, unstable angina, need for intervention with CABG or angioplasty, or the frequency and severity of angina pectoris. Nor was there a significant difference between the groups with respect to ischaemic neurological events. The single most common reason for withdrawal from blind treatment was the need for open treatment with $\beta$ blockade. Significantly more patients in the placebo group withdrew as a result of need for open treatment with $\beta$
Table 6 Findings at exercise two years after coronary artery bypass grafting

\begin{tabular}{|c|c|c|c|c|c|}
\hline & \multicolumn{2}{|c|}{ Placebo } & \multicolumn{2}{|c|}{ Metoprolol } & \multirow[b]{2}{*}{ Pvalue } \\
\hline & $n$ & $\%$ & $n$ & $\%$ & \\
\hline \multicolumn{6}{|l|}{$\begin{array}{l}\text { Reason for termination } \\
\text { of exercise }(2)^{\mathrm{a}}\end{array}$} \\
\hline General tiredness & 151 & 49 & 153 & 50 & \\
\hline Leg tiredness & 63 & 20 & 65 & 21 & \\
\hline Chest pain & 10 & 3 & 18 & 6 & \\
\hline Fall in systolic blood pressure & 5 & 2 & 6 & 2 & \\
\hline ST depression & 6 & 2 & 4 & 1 & \\
\hline Dyspnoea & 38 & 12 & 32 & 11 & \\
\hline Claudication & 7 & 2 & 14 & 5 & \\
\hline Muscular or neurological & 6 & 2 & 6 & 2 & \\
\hline Other & 24 & 8 & 8 & 3 & 0.006 \\
\hline \multicolumn{6}{|l|}{ Arrhythmia during } \\
\hline $\begin{array}{l}\text { exercise }(17)^{\mathrm{a}} \\
\text { Supraventricular extrasystoles }\end{array}$ & 182 & 60 & 144 & 48 & 0.004 \\
\hline $\begin{array}{l}\text { Supraventricular extrasystoles } \\
\text { Total }\end{array}$ & 71 & 23 & 64 & 21 & \\
\hline$>5 / \mathrm{min}$ & 13 & 4 & 7 & 2 & \\
\hline Ventricular extrasystoles & & & & & \\
\hline Total & 158 & 52 & 121 & 41 & 0.005 \\
\hline$>5 / \mathrm{min}$ & 57 & 19 & 38 & 13 & 0.04 \\
\hline \multicolumn{6}{|l|}{ Medication } \\
\hline Still on blind treatment & 201 & 65 & 224 & 73 & 0.03 \\
\hline$\beta$ blockade, but not blind treatment & 64 & 21 & 37 & 12 & 0.004 \\
\hline Calcium antagonists & 39 & 13 & 34 & 11 & \\
\hline Long acting nitrates & 25 & 8 & 25 & 8 & \\
\hline Diuretics for CHF & 48 & 15 & 39 & 13 & \\
\hline Digitalis & 40 & 13 & 19 & 6 & 0.006 \\
\hline Other CHF treatment & 12 & 4 & 7 & 2 & \\
\hline Other antihypertensives & 37 & 12 & 23 & 8 & \\
\hline
\end{tabular}

CHF, congestive heart failure.

${ }^{a}$ Numbers in parentheses denote missing patients.

Table 7 Clinical events and reasons for withdrawal from blind medication postoperatively

\begin{tabular}{|c|c|c|c|c|c|}
\hline & \multicolumn{2}{|c|}{$\begin{array}{l}\text { Placebo } \\
(n=311)\end{array}$} & \multicolumn{2}{|c|}{$\begin{array}{l}\text { Metoprolol } \\
(n=307)\end{array}$} & \multirow[b]{2}{*}{$P$ value } \\
\hline & $n$ & $\%$ & $n$ & $\%$ & \\
\hline $\begin{array}{l}\text { Cardiac events } \\
\text { Myocardial infarction } \\
\text { Unstable angina } \\
\text { Need for CABG } \\
\text { Need for PTCA }\end{array}$ & $\begin{array}{l}4 \\
7 \\
4 \\
4\end{array}$ & $\begin{array}{l}1 \cdot 3 \\
2 \cdot 3 \\
1 \cdot 3 \\
1 \cdot 3\end{array}$ & $\begin{array}{l}3 \\
9 \\
4 \\
3\end{array}$ & $\begin{array}{l}1 \cdot 0 \\
2 \cdot 9 \\
1 \cdot 3 \\
1 \cdot 0\end{array}$ & \\
\hline $\begin{array}{l}\text { Neurological events } \\
\text { Transitory ischaemic attack } \\
\text { Stroke }\end{array}$ & $\begin{array}{l}2 \\
6\end{array}$ & $\begin{array}{l}0.6 \\
1.9\end{array}$ & $\begin{array}{l}3 \\
5\end{array}$ & $\begin{array}{l}1 \cdot 0 \\
1.6\end{array}$ & \\
\hline $\begin{array}{l}\text { Angina functional class } \\
\text { two years postoperatively }(24)^{a} \\
1 \\
2 \\
3 \\
4\end{array}$ & $\begin{array}{r}228 \\
50 \\
20 \\
3\end{array}$ & $\begin{array}{r}76 \\
17 \\
7 \\
1\end{array}$ & $\begin{array}{r}222 \\
43 \\
28 \\
0\end{array}$ & $\begin{array}{r}76 \\
15 \\
10 \\
0\end{array}$ & \\
\hline $\begin{array}{l}\text { Reasons for withdrawal } \\
\text { from blind medication } \\
\text { within two years } \\
\text { Clinical event } \\
\text { Need for open treatment with } \beta \text { blockade } \\
\text { Hypotension } \\
\text { Bradycardia } \\
\text { Signs of poor peripheral circulation } \\
\text { Other physical or psychological reason } \\
\text { Other }\end{array}$ & $\begin{array}{r}13 \\
58 \\
4 \\
1 \\
6 \\
23 \\
22\end{array}$ & $\begin{array}{c}4 \\
19 \\
1 \\
0 \cdot 3 \\
2 \\
7 \\
7\end{array}$ & $\begin{array}{r}13 \\
20 \\
6 \\
7 \\
1 \\
18 \\
26\end{array}$ & $\begin{array}{l}4 \\
7 \\
2 \\
2 \\
0 \cdot 3 \\
6 \\
8\end{array}$ & $\begin{array}{l}<0.0001 \\
0.04\end{array}$ \\
\hline
\end{tabular}

CABG, coronary artery bypass grafting; PTCA, percutaneous transluminal coronary angio-

${ }^{\text {plasty. }}$ Number in parentheses denotes missing patients; 'In all, 15 of the patients had more than one reason for withdrawal. blockade: $19 \%$ versus $7 \%$ in the metoprolol group $(\mathrm{P}<0.0001)$. Bradycardia was more often a reason for withdrawal in the metoprolol group $(P=0.04)$.

\section{Discussion}

This study describes exercise capacity and signs of myocardial ischaemia at exercise two years after CABG in a double blind randomised study of metoprolol. No significant difference in working capacity, functional improvement, ST segment depression, or the presence of angina was found between the groups.

\section{LIMITATIONS}

\section{Patient selection}

The patients participating in the present analysis represent $26 \%$ of the patients who underwent CABG during the study period. Patients in need of $\beta$ blockade for clinical reasons and patients with an intolerance to $\beta$ blockade were excluded from participation in the main study. The participants therefore represent a selected group of patients. Whether the findings in this study can be extrapolated to an unselected group of patients can be questioned.

Furthermore, only two thirds of the patients participating in the metoprolol trial performed a two year exercise test. The reasons for omission were not recorded, although in $42 \%$ it was attributed to lack of systematic follow up at two centres. The baseline data for non-participants show more advanced heart disease in this group, thereby representing a selection towards healthier patients performing exercise tests. The omission of the more severely diseased patients decreases the sensitivity of the study. However, this kind of selection is likely to be present in all exercise test studies.

In this analysis, $31 \%$ of the patients were withdrawn from blind treatment prematurely. More patients in the placebo group withdrew prematurely compared with the metoprolol group, thereby creating difficulties in the interpretation of the data. This was largely explained by open treatment with $\beta$ blockade in the placebo group: $21 \%$ compared with $12 \%$ in the metoprolol group ( $P=0.004)$. We therefore expect the patients in greatest need of therapy with metoprolol to have received this openly, thereby reducing the possibility of detecting any differences between groups.

Electrocardiographic technique and study design Several ECG recording systems were used, making the interpretation of the data more complex. The decision to record only leads aVF, V2, and V5 may have decreased the sensitivity of the method. The exercise tests were performed at the referring hospital and mailed to the investigators. The exercise test participation and the completeness of the protocol data should have been even better, had the principal investigators been directly responsible for the testing. No correction was made for multiple comparisons in the statistical 
evaluation, and $P$ values with a low degree of significance must be regarded with caution.

EXERCISE CAPACITY, ST SEGMENT DEPRESSION, AND ISCHAEMIA

A general improvement in exercise capacity after CABG, like that found in our study population, has been well established in earlier studies. ${ }^{112}$ Other investigators have found that $\beta$ blockade after acute myocardial infarction does not influence maximum work load. ${ }^{1314}$ In stable angina pectoris, metoprolol treatment has been shown to increase exercise duration and decrease ST level depression. ${ }^{15} 16$ Treatment with $\beta$ blockers in hypertensive patients ${ }^{17}$ and healthy volunteers has revealed impaired endurance capacity, ${ }^{18}$ whereas treatment in patients with left ventricular dysfunction has shown improved exercise capacity. ${ }^{19} 20$ This latter phenomenon is in line with a beneficial effect by metoprolol on left ventricular function in dilated cardiomyopathy. ${ }^{21}$

ST segment depression after myocardial infarction has been shown to be less frequent after treatment with metoprolol at submaximum exercise. ${ }^{22}$ However, in a patient group with suspected myocardial infarction, ST depression occurred at a similar frequency among patients treated with metoprolol, as compared to placebo, at exercise. ${ }^{23}$

There was a trend towards better performance in the metoprolol group, but the difference was not significant. There was also a trend, by ST depression criteria and by physician's assessment, indicating less myocardial ischaemia among patients randomised to metoprolol. The clinical data suggest that the patients who refrained from exercise testing were at higher risk, and this may have been a group which would have benefitted from $\beta$ blockade. It is possible that if all of the potential study group had been exercised such a change would have been confirmed. However, any speculations about a beneficial effect of $\beta$ blockade were offset by the observations of a trend towards higher prevalence of chest pain adjudged as angina (NS), and a higher mean chest pain score in the metoprolol group $(P=0.001)$.

As in other studies, the presence of ST segment changes-assessed as silent myocardial ischaemia-after CABG was considerable (table 4). ${ }^{24}$ There appeared to be more angina pectoris in the metoprolol group and more silent myocardial ischaemia in the placebo group, which was unexpected since earlier studies have revealed less pronounced symptoms during exercise with metoprolol treatment for angina pectoris. ${ }^{15}$ The possibility that patients in the metoprolol group reached a higher work load was obviously not the explanation. Since patients displaying chest pain adjudged as angina pectoris represented only approximately $13 \%$ of all those participating in postoperative exercise tests, and mean chest pain in both treatment groups was close to pain score 1 according to the Borg scalewhich corresponds to chest pain classified as "very weak" - we are doubtful about the relevance of this finding. In table 4 , fewer patients were adjudged to actually show definite or probable myocardial ischaemia than the number of patients who fulfilled the ECG criteria for myocardial ischaemia. This difference was partly explained by the fact that the ECG criteria rigorously specified ST depression of $\geqslant 1 \mathrm{~mm}$, and did not account for the presence of, for example, signs of left ventricular hypertrophy, bundle branch block, other ST depression at rest, $Q$ waves, $Q S$ complexes, or pacemaker rhythm in the resting ECG. The value of ST segment depression during exercise in the diagnosis of myocardial ischaemia after CABG has been questioned. ${ }^{25} 26$ However, the prognosis has been shown to correlate with ST segment depression and functional improvement. ${ }^{24}$ The best exercise performance and the least ST segment depression have been observed in patients with the most complete revascularisation. ${ }^{27} 28$ When the exercise tests were analysed in relation to the actual treatment with $\beta$ blockade, we found a difference in favour of no treatment. One reason for this may be that among patients withdrawn from blind medication and treated with open $\beta$ blockade, a large proportion actually had myocardial ischaemia. They were expected to have a poorer exercise performance. Another explanation may be that asymptomatic participants randomised to metoprolol treatment two years after CABG are very similar to patients without coronary artery disease and are thus expected to perform less well on $\beta$ blockade treatment.

In summary, patients who have undergone CABG constitute a complex group in terms of the degree of revascularisation to the myocardium, left ventricular function, and the presence of ischaemic symptoms, which may in part explain the lack of difference between the treatment groups in terms of exercise capacity and occurrence of ST segment depression.

\section{OTHER FINDINGS}

Systolic blood pressure and heart rate at exercise were higher in the placebo group, thereby corresponding with previous studies on the effect of $\beta$ blockade in stable angina ${ }^{1516}$ and after myocardial infarction. ${ }^{29}$

The higher prevalence of ventricular premature beats in the placebo group was in agreement with previous experience of patients treated with $\beta$ blockade after myocardial infarction. ${ }^{1430}$ However, ventricular ectopy after CABG has not been found to be related to prognosis, ${ }^{12} 31$ thus indicating that this effect is of lesser importance. A considerable proportion of patients in both treatment groups had ventricular premature beats during exercise, just above half in the placebo group versus $41 \%$ in the metoprolol group $(P=0.005)$. We therefore chose to present data for more than five ventricular premature beats per minute, in order to evaluate if the group differences remained or increased. Occurrence of several ventricular premature beats was also less frequent in the metoprolol group, although the difference was only of borderline significance $(P=0.04$; table 6$)$. The greater use of digitalis in the placebo 
group (table 6) may be explained by at least two factors: (1) the higher heart rate with placebo treatment, may, in combination with dyspnoea, have constituted the basis for treatment in some cases; (2) we cannot exclude the possibility of a beneficial effect on left ventricular function caused by metoprolol. ${ }^{21}$

The single most important reason for withdrawal was the need for open treatment with $\beta$ blockade, which was significantly more common in the placebo group. These numbers are somewhat lower than the numbers in table 6 , which refers to patients who were on treatment with $\beta$ blockade at the exercise test two years after CABG. The difference is because some patients were withdrawn for reasons other than the need for $\beta$ blockade, but were prescribed this treatment later.

The clinical neurological and cardiac event rate was comparable between the two groups, supporting the comparability of the two treatment groups.

\section{IMPLICATIONS}

Treatment with metoprolol for two years after CABG did not significantly reduce the electrocardiographic signs of myocardial ischaemia on effort, nor did it increase exercise capacity. The present study does not therefore support the use of $\beta$ blockade as routine prophylactic treatment after CABG. Treatment should be given only according to accepted clinical indications. A trend towards better exercise performance and less myocardial ischaemia on metoprolol two years after surgery was observed. The withdrawal rate and the omission from two year exercise testing were considerable, occurring foremost among the patients at highest risk, who would also have the best chance of benefitting from $\beta$ blockade. We cannot exclude the possibility of subgroups, such as patients with impaired left ventricular function or signs of immediate postoperative myocardial ischaemia, who might benefit from metoprolol treatment.

The authors are grateful to Thomas Karlsson MSc for his expert statistical contribution, Lisbeth Winberg RA for her skilled assistance with the exercise tests, and Maria Haglid RA for her meticulous administration of patients. We should like to thank the following colleagues who were responsible for the performance of exercise tests outside Göteborg: Alingsås performance of exercise tests outside Göteborg: Alingsås Rosqvist MD; Kungälv Hospital, Gun Larsson MD; Lidköping Hospital, Gunnar Stenström MD PhD; Lysekil Hospital, Peter Hoffman MD; Skene Hospital, Arne Rindner MD; Skövde Hospital, Jan Ejdebäck MD PhD; Strömstad Hospital, Fritiof Wandel MD; Säffle Hospital, Lennart Gillgren MD; Trollhättan Hospital,
Varberg Hospital, Carl Nemeczek MD.

1 Myers WO, Schaff HV, Gersh BJ, Fisher LD, Kosinski AS, Mock MB, et al. Improved survival of surgically treated patients with triple vessel coronary artery disease and severe angina pectoris. $\mathcal{F}$ Thorac Cardiovasc Surg and severe angina

2 Califf RM, Harrell FE, Lee KL, Rankin JS, Hlatky MA, Mark DB, et al. The evolution of medical and surgical Mark DB, et al. The evolution of medical and surgical therapy for coronary artery dis
tive. $₹ A M A$ 1989;261:2077-86.

3 Smith SH, Geer JC. Morphology of saphenous vein-coronary artery bypass grafts. Arch Pathol Lab Med 1983; 107:13-8.

4 Campeau L, Enjalbert M, Lespérance J, Bourassa MG, Kwiterovich $\mathrm{P}$, Wacholder $S$, et al. The relation of risk factors to the development of atherosclerosis in saphenous-vein bypass grafts and the progression of disease in the native circulation. $N$ Engl $\mathcal{F}$ Med 1984;311:1329-32.
Kaplan JR, Manuck SB, Adams MR, Clarkson TB. The effects of beta-adrenergic blocking agents on atherosclerosis and its complications. Eur Heart $\Im$ 1987;8:928-44.

6 Angelhed J-E, Bjurö TI, Ejdebäck J, Selin K, Schlossman
D, Griffith LSC, et al. Computer aided exercise electrocardiographic testing and coronary arteriography in cardiographic testing and coronary arteriography in patients with angina pectoris and

7 Pahlm O, Sörnmo L. Data processing of exercise ECG's. IEEE Trans Biomed Eng 1987;34:158-65.

8 Borg GAV. Psychophysical bases of perceived exertion. Med Sci Sports Exerc 1982;14:377-81.

9 Wagner NB, Sevilla DC, Krucoft MW, Lee KL, Pieper $\mathrm{KS}$, Kent KK, et al. Transient alterations of the QRS complex and ST segment during percutaneous transluminal ballon angioplasty of the left anterior descending coronary artery. Am $\mathcal{f}$ Cardiol 1988;62:1038-42.

10 Froelicher VF. Manual of exercise testing, 2nd ed. St Louis: CV Mosby, 1994:22.

11 European Coronary Surgery Study Group. Long-term results of prospective randomised study of coronary results of prospective randomised study of coronary artery bypass surge

12 Hultgren HN, Peduzzi P, Detre K, Takaro T. The 5 year effect of bypass surgery on relief of angina and exercise performance. Circulation 1985;72(suppl V):79-83.

13 Rønnevik PK, Gundersen T, Abrahamsen AM. Long-term effect of beta-blockade with timolol on maximal work capacity following myocardial infarction. Eur Heart $\mathcal{f}$ 1983;4:773-80.

14 Olsson G, Rehnqvist N, Freyschuss U, Zetterqvist S. Influence of long-term metoprolol treatment on early and late exercise test performance after acute myocardial infarction. $\mathrm{Am}$ F Cardiol 1988;61:519-23.

15 Thadani U, Davidson C, Singleton W, Taylor SH. Comparison of five beta-adrenoreceptor antagonists with different ancillary properties during sustained twice daily therapy in angina pectoris. Am $\mathcal{H}$ Med 1980;68: daily therat 2430 .

16 van Dijk RB, Lie KI, Crijns HJGM. Diltiazem in comparison with metoprolol in stable angina pectoris. Eur Heart $f$ 1988;9:1194-9.

17 van Baak MA, Böhm ROB, Arends BG, van Hooff MEJ, Rahn $\mathrm{KH}$. Long-term antihypertensive therapy with beta-blockers: submaximal exercise capacity and metabolic effects during exercise. Int $\mathcal{F}$ Sports Med 1987;8: 342-7.

18 Lundborg P, Åström H, Bengtsson C, Fellenius E, von Schenck H, Svensson L, et al. Effect of beta-adrenoceptor blockade on exercise performance and metabolism. Clin Sci 1981;61:299-305.

19 Engelmeier RS, O'Connell JB, Walsh R, Rad N, Scanlon PJ, Gunnar RM. Improvement in symptoms and exercise tolerance by metoprolol in patients with dilated cardiomyopathy: a double-blind, randomized, placebocardiomyopathy: a double-blind, randomized,

20 Andersson B, Blomström-Lundqvist C, Hedner T, Waagstein F. Exercise hemodynamics and myocardial metabolism during long-term beta-adrenergic blockade metabolism during long-term beta-adrenergic blockade
in severe heart failure. $₹ \mathrm{Am}$ Coll Cardiol 1991;18: in severe

21 Waagstein F, Bristow MR, Swedberg K, Camerini F, Fowler MB, Silver MA, et al. Beneficial effects of metoprolol in idiopathic dilated cardiomyopathy. Lancet 1993;342:1441-6.

22 Ades PA, Thomas JD, Hanson JS, Shapiro SM, LaMountain J. Effect of metoprolol on the submaximal stress test performed early after acute myocardial infarction. Am f Cardiol 1987;60:963-6.

23 Rønnevik PK, Gundersen T, Abrahamsen AM, Knutsen $\mathrm{H}$, Woie L. Effects of metoprolol on early exerciseinduced ST-segment changes and ventricular arrhythmias in patients with suspected acute myocardial mias in patients with suspected
infarction. Int $\mathcal{F}$ Cardiol 1989;22:51-7.

24 Weiner DA, Ryan TJ, Parson L, Fisher LD, Chaitman BR, Sheffield LT, et al. Prevalence and prognostic significance of silent and symptomatic ischemia after coronary bypass surgery: A report from the coronary artery surgery study (CASS) randomized population. $₹ \mathrm{Am}$ Coll Cardiol 1991;18:343-8.

25 Dubach P, Froelicher V, Klein J, Detrano R. Use of the exercise test to predict prognosis after coronary artery bypass grafting. Am $\mathcal{F}$ Cardiol 1989;63:530-3.

26 Visser FC, van Campen L, de Feyter PJ. Value and limitations of exercise stress testing to predict the functional results of coronary artery bypass grafting. Int $\mathcal{F}$ Cardiac Imaging 1993;9:41-7.

27 Gohlke H, Gohlke-Bärwolf C, Samek L, Sturzenofecker P, Schmuziger M, Roskamm H. Serial exercise testing up to 6 years after coronary bypass surgery: behavior of exercise parameters in groups with different degrees of revascularization determined by postoperative angiograrevascularization determined by po
phy. Am $\mathcal{f}$ Cardiol 1983;51:1301-6.

28 Frick MH, Harjola P-T, Valle M. Persistent improvement after coronary bypass surgery: ergometric and angiographic correlations at 5 years. Circulation 1983;67: graphic

29 Curtis JL, Houghton JL, Patterson JH, Koch G, Bradley DA, Adams KF. Propranolol therapy alters estimation of potential cardiovascular risk derived from submaximal postinfarction testing. Am Heart $\mathcal{f}$ 1991;121:1655-64.

30 Reynolds JL, Whitlock RML. Effects of a beta-adrenergic receptor blocker in myocardial infarction treated for one receptor blocker in myocardial infarction tre
year from onset. $B r$ Heart $₹ 1972 ; 34: 252-9$.

31 Yli-Mäyry S, Huikuri HV, Korhonen UR, Airaksinen KEJ, Ikäheimo MJ, Linnaluoto MK, et al. Prevalence and prognostic significance of exercise-induced ventricular arrhythmias after coronary artery bypass grafting. $\mathrm{Am} \mathcal{F}$ Cardiol 1990;66:1451-4. 